\title{
Comparison of intermittent pneumatic compression device and compression stockings for workers with leg edema and pain af- ter prolonged standing
}

\author{
Da-Sol Kim 1,2, Yu Hui Won 1,2,* and Myoung-Hwan Ko 1,2,* \\ 1 Department of Physical Medicine and Rehabilitation, Jeonbuk National University Medical School, Jeonju, \\ Republic of Korea; murunoon@google.com (DSK) \\ 2 Research Institute of Clinical Medicine of Jeonbuk National University-Biomedical Research Institute of \\ Jeonbuk National University Hospital, Jeonju, Republic of Korea \\ * Correspondence: mhko@jbnu.ac.kr (MHK), wonyh@jbnu.ac.kr (YHW) Tel.: +82-250-1820
}

\begin{abstract}
During prolonged standing, insufficient calf muscle pumping accompanies venous stasis and hypertension in the lower legs, resulting in valve dysfunction, venous wall problems, and subsequent inflammation. Compression therapy, which includes medical compression stockings (MCS) and mechanical intermittent pneumatic compression (IPC), is one of the most effective therapeutic interventions for treating chronic venous diseases. This study aimed to investigate the safety and efficacy of IPC and MCS, and the optimal protocol of compression therapy for chronic venous disease. This crossover trial was conducted for healthy, long standing workers ( $>8 \mathrm{~h}$ daily) with leg edema and pain. Four groups were established for each visit: group A (resting after work without MCS), group B (resting after work with MCS), group C (IPC after work without MCS), and group $\mathrm{D}$ (IPC after work with MCS). The primary outcome was the visual analogue scale (VAS) score for leg pain. The secondary outcomes were leg volume $(\mathrm{mL})$, circumference $(\mathrm{cm})$, extracellular fluid/total body fluid (ECF/TBF), and extracellular water/total body water (ECW/TBW) through bioimpedance analysis. Outcomes were assessed before work (T0), after work (T1), and 60 minutes after intervention (T2). We included 39 healthy volunteers, aged 30.03 \pm 7.56 years. All four groups had significantly increased leg pain after work (T0-1) but improved 60 minutes after intervention (T1-2), particularly group C (decreased VAS by 1.9). When leg swelling was compared at $\mathrm{T} 0$ and $\mathrm{T} 1$, groups $A$ and $C$ showed significant increases in leg volume and circumference, indicating significant workinduced edema, whereas groups $B$ and $D$ showed no change or even a decrease. After interventions, leg volume and circumference significantly decreased in groups $A$ and $C$, although groups $B$ and $C$ did not show significant improvement. The ECF/TBF and ECW/TBW of all groups decreased after interventions. Leg pain and edema after prolonged standing in healthy adults were safely and effectively improved by IPC. Although MCS also reduced leg edema immediately after work, it did not show significant improvement in leg pain at T0-1-and T1-2, nor in swelling at T1-2.
\end{abstract}

Keywords: Intermittent Pneumatic Compression Devices; Compression Stocking; Edema; Pain, Standing

\section{Introduction}

Many workers are required to stand for long periods of time, these workers include nurses, teachers, shop assistants, cooks, pharmacists, and hairdressers, or sit, such as office workers, drivers, and information technology specialists [1,2]. In Dutch standing work guidelines, more than $1 \mathrm{~h}$ of continuous standing and more than $4 \mathrm{~h}$ of standing in total is unsafe and places the individual at a high risk of strain and health problems, and for which immediate intervention to reduce the strain should be performed [3]. Prolonged standing has reportedly been associated with negative health outcomes, including chronic 
venous diseases, fatigue, low back pain, carotid atherosclerosis, ischemic heart disease, orthostatic intolerance, and pregnancy issues, such as increased preterm birth or spontaneous abortion [4]. Leg immobility from prolonged standing causes ineffective calf muscle pumping and subsequent venous blood pooling and hypertension in the lower extremities [5]. These hemodynamic abnormalities in the veins of the lower legs might be related to valve dysfunction, increased venous wall tension and distension, and endothelial dysfunction and leukocyte infiltration, which lead to a cascade of inflammation [5].

Prolonged standing commonly contributes to a physiologic venous insufficiency in healthy long-standing workers, who complain of swelling of the lower limbs, a heavy feeling in the legs, leg numbness, skin itching, dilated capillaries, varicose veins, and skin discoloration [2]. At the same time, the prevalence of pathologic chronic venous insufficiency (CVI) is higher in this group. Previous studies on 636 healthy healthcare workers suggested that $69 \%$ of hospital employees presented with clinical signs of CVI (C $\geq 1$ of the Clinical, Etiology, Anatomy, and Pathophysiology [CEAP] classification), and 82\% of them with newly detected venous reflux on ultrasound [1].

Treatment of venous disease consists of medication and conservative treatment, including compression by elastic stockings, bandage, and intermittent pneumatic pressure; sclerotherapy; endovenous ablation; cyanoacrylate; and surgical intervention [6, 7]. Rabe et al. demonstrated that all levels of medical compression stockings (MCS), which is the most common form of compression therapy, are recommended for the improvement of occupational leg venous symptoms, quality of life, and edema [8]. Mechanical intermittent pneumatic compression (IPC) using pneumatic cuffs connected to a pump is used for limb compression therapy. IPC has been used for the prevention of deep vein thrombosis and the treatment of arterial disease, lymphedema, and chronic venous insufficiency, especially venous ulcers. Although both MCS and IPC have long histories of clinical application, there is no standard consensus on the frequency, pressure, duration, and combination protocol with other methods of compression and surgical intervention.

The aim of this study was to compare four protocols of compression therapy (natural resting or IPC with or without MCS during work time) in the relief of venous symptoms of prolonged stationary healthy subjects. The primary outcome was the subjective pain score (visual analogue scale), and the secondary outcomes were the objective measures of leg volume and circumference. These results can provide the optimal protocol of compression therapy for the improvement of venous symptoms in prolonged stationary healthy subjects.

\section{Materials and Methods}

\subsection{Study population}

We recruited healthy adults aged 19 years and older who worked in a prolonged stationary standing position at the Biomedical Research Institute and Translational Research and Clinical Trial Center for Medical Device, Jeonbuk National University, from December 2019 to April 2020. According to the "Health Guide for People who Work Standing" published by the Korea Occupational Safety and Health Agency, "jobs that require prolonged standing include salespersons or cashiers at wholesale and retailers such as large discount stores, launderers, hairdressers, workers in assembly lines, packaging industry, construction workers, healthcare employees, teachers" [9]. The inclusion criteria were: 1) current working history of prolonged standing for $\geq 8 \mathrm{~h}$ per day; 2 ) having self-reported venous symptoms, such as leg pain and swelling; and 3) fully understanding the purpose and procedures of the study, and voluntarily expressing willingness to participate. The exclusion criteria were: 1) younger than 19 years old; 2) cognitive impairment with difficulty in expressing the pain location and scoring using the analogue scale by themselves; 3) ankle 
brachial index $\leq 0.8$, with suspected underlying peripheral arterial occlusive disease; 4) diagnosis of deep vein thrombosis and other venous obstructive disorders on duplex ultrasound during the screening visit; 5) leg hypoesthesia; 6) recent leg surgery (within 6 months); and 7) other individually applied criteria at the discretion of the investigator. Ethical approval for the study was obtained from the institutional review board of Jeonbuk National University Hospital (CUH 2019-05-064-002), and written informed consent was obtained from all participants.

\subsection{Study stocking and device}

\subsubsection{Standard medical compression stocking}

The Stocking Simply Coton Fin (THUASNE, Levallois-Perret, France) is a commonly prescribed thigh-high MCS with closed toes exerting 23-32 mmHg pressure at the medial supramalleolar area.

\subsubsection{Intermittent pneumatic compression device}

We used an SMA-100 (WelbuTech, Seoul, Korea), an IPC device with five chambers which can change its pressure ranging from 0 to $200 \mathrm{mmHg}$. Identical pressure was applied to all chambers; the pneumatic compression shifts to the next chamber once the set pressure has been reached inside the previous chamber, as measured by a pressure sensor. There are two IPC modes of application (sequential and circular modes). In the sequential mode, the set pressure is initially pumped into the distal chamber; after the distal chamber is deflated, the pressure is pumped into the proximal chamber. On the other hand, in the circular mode, the distal chamber is pumped followed by the proximal chamber, without decompression of the distal chamber. After all chambers are inflated, the chambers are deflated at once.

\subsection{Study design}

This study was a prospective crossover clinical trial conducted. People who worked in a prolonged stationary standing position and complained of leg pain and swelling were screened. After assessing eligibility and obtaining written informed consent, participants were screened according to the inclusion and exclusion criteria (Figure 1). Except for one subject who was lost to follow-up, 39 participants enrolled in the study following the screening completed five study visits and were categorized into four occupational groups: part-time lecturer, teacher, cashier, and healthcare worker (mostly nurse). During the screening visit, we collected demographic data, baseline vital signs, medical histories (past histories and chronic underlying diseases), and current medications, and performed physical examination, duplex ultrasound, and ankle-brachial index test. For visits 1 to 4 , measurements of leg pain (using the visual analogue scale [VAS]), leg volume, circumference, and bioelectrical impedance analysis (BIA) were performed three times during each of the study visits (T0: morning visit before work, T1: evening visit after work, T2: 60 min after natural resting or interventions). For visit 1 , participants worked in a standing position without wearing MCS and rested for 60 minutes in the supine position (Group A). For visit 2 (within 7 days of visit 1), MCS was used for more than $8 \mathrm{~h}$ during the workday and naturally rested in the same manner as in visit 1 (Group B). At visit 3, they did not wear MCS and used the IPC device for a total of $60 \mathrm{~min}$, with $30 \mathrm{~min}$ of circular and sequential modes at a pressure of $90-130 \mathrm{mmHg}$ in the supine position (Group C). Finally, for visit $4, \mathrm{MCS}$ was worn for more than $8 \mathrm{~h}$ during the workday and the IPC device was used 
similarly as in visit 3 (Group D). The pressure in each chamber was set by the participants within the range of $90-130 \mathrm{mmHg}$, such that the pressure did not cause excessive pain or discomfort. A total of nine visits were required, including the screening visit and visits 1 to 4 (within 7 days of the previous visit, comprising of 2 visits each: before and after work). Participants had to engage in work that required at least $8 \mathrm{~h}$ of standing and were asked to refrain from any activity that may reduce leg pain and swelling during work, for example resting on their backs. Participants were monitored for safety issues and adverse events. This trial protocol was registered at the Clinical Research Information Service (KCT0005383).

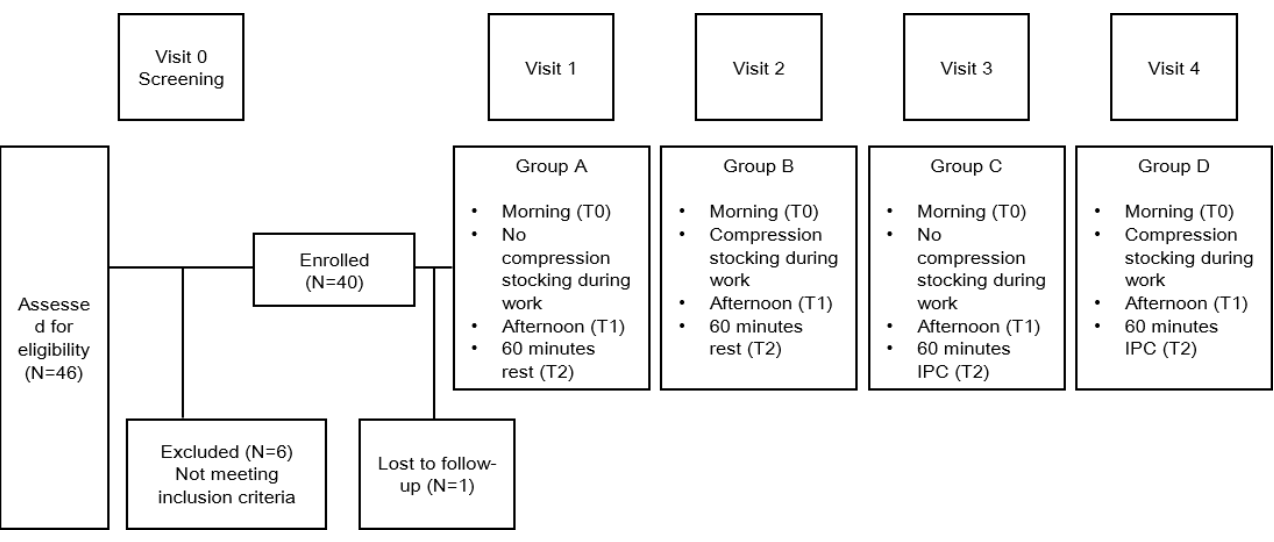

Figure 1. Flowchart of the procedures for the clinical trial

\subsection{Outcome measures}

\subsubsection{Primary outcome}

The primary outcome was leg pain evaluated via VAS, which was a continuous scale, defined as 0 for no pain and 10 for the most severe pain.

\subsubsection{Secondary outcomes}

The secondary outcome was leg swelling as measured by leg volume, circumference, and BIA. We measured leg volume in each leg using the water displacement method and recorded it in milliliters [10]. Leg circumference was also measured in each leg, with the limb in a relaxed position. We measured the circumference of each foot, ankle at $2 \mathrm{~cm}$ above the medial malleolus, calf at $10 \mathrm{~cm}$ below the inferior pole of the patella, distal thigh at 10 $\mathrm{cm}$ above the superior pole of the patella, and proximal thigh at $20 \mathrm{~cm}$ above the superior pole of the patella [11]. For BIA, we evaluated extracellular fluid (ECF), total body fluid (TBF), extracellular water (ECW), and total body water (TBW) using the Inbody 3.0 system (Biospace Co., Seoul, South Korea), which provides whole body, trunk, torso, and limb values [12,13]. Participants stood on the Inbody 720 scale with their sole in contact with the foot electrodes as motionless as possible. Then, they grasped the handle with each palm, finger, and thumb making contact with the hand electrodes. 


\subsubsection{Statistical analysis}

All statistical analyses were conducted using the SPSS 24.0 software. For each study visit, the primary outcome (VAS) and secondary outcomes (leg volume and leg circumference) were compared across $\mathrm{T} 0, \mathrm{~T} 1$, and $\mathrm{T} 2$. We compared the primary and secondary outcomes between $\mathrm{T} 0$ and $\mathrm{T} 1$ in each group to identify the provocation of leg pain and swelling after work in the prolonged standing position, and between T1 and T2 to verify the effect of the interventions. Normality was tested using the Shapiro-Wilk test. Normally distributed data were analyzed with a paired t-test or repeated measures analysis of variance (RMANOVA) followed by a post-hoc test to determine whether significant differences occurred with respect to treatment interventions. Non-normally distributed data were analyzed with the nonparametric Friedman test followed by a Wilcoxon signed rank test to determine whether significant differences occurred with respect to treatment interventions. Bonferroni correction was performed for the post-hoc test or Wilcoxon signed rank test to ensure that the alpha was maintained at 0.05 .

\section{Results}

\subsection{Subject demographics}

A total of 46 healthy subjects were eligible for study participation, of whom 40 were enrolled in the study and 6 were excluded because they did not meet the inclusion criteria. Only one participant was lost to follow-up, and 39 participants completed the final visit. The baseline characteristics are presented in Table 1. Of the 39 participants, the mean age was 30.03 (range, 22 -53) years, and 51.3\% were female. Most subjects were employed in the following occupations: part-time lecturers, 10 subjects $(25.6 \%)$; teachers, 9 subjects $(23.0 \%)$; cashiers, 10 subjects (25.6\%); and healthcare workers, 10 subjects $(25.6 \%)$. At the screening ultrasound examination, 35 of the 39 participants had newly detected deep vein reflux and no one had deep vein thrombosis or other obstructive arteriovenous diseases. In addition, any peripheral arterial obstruction was discovered in the ABI test. The 35 participants with detected venous reflux and no arterial disorder on imaging studies were diagnosed with chronic venous disease class C3 (edema) according to the CEAP classification.

\subsection{Pain score as the primary outcome}

In all groups, the pain score measured in the afternoon after work (T1) significantly increased by more than 1.5 compared to that measured in the morning before work (T0), showing that leg pain was provoked at T1 by prolonged standing work (Table 2, Figure 2 ). After four interventions, the differences in the scores between the post-workday and post-intervention periods (T1-T2) were significant in all groups with respect to pain reduction. There were also significant differences among the four interventions. The posthoc test showed differences in pain improvement between A and C, A and D, B and C, and $B$ and $D$, but we did not observe any differences between $A$ and $B$, and $C$ and $D$ (Supplementary Table S1). 


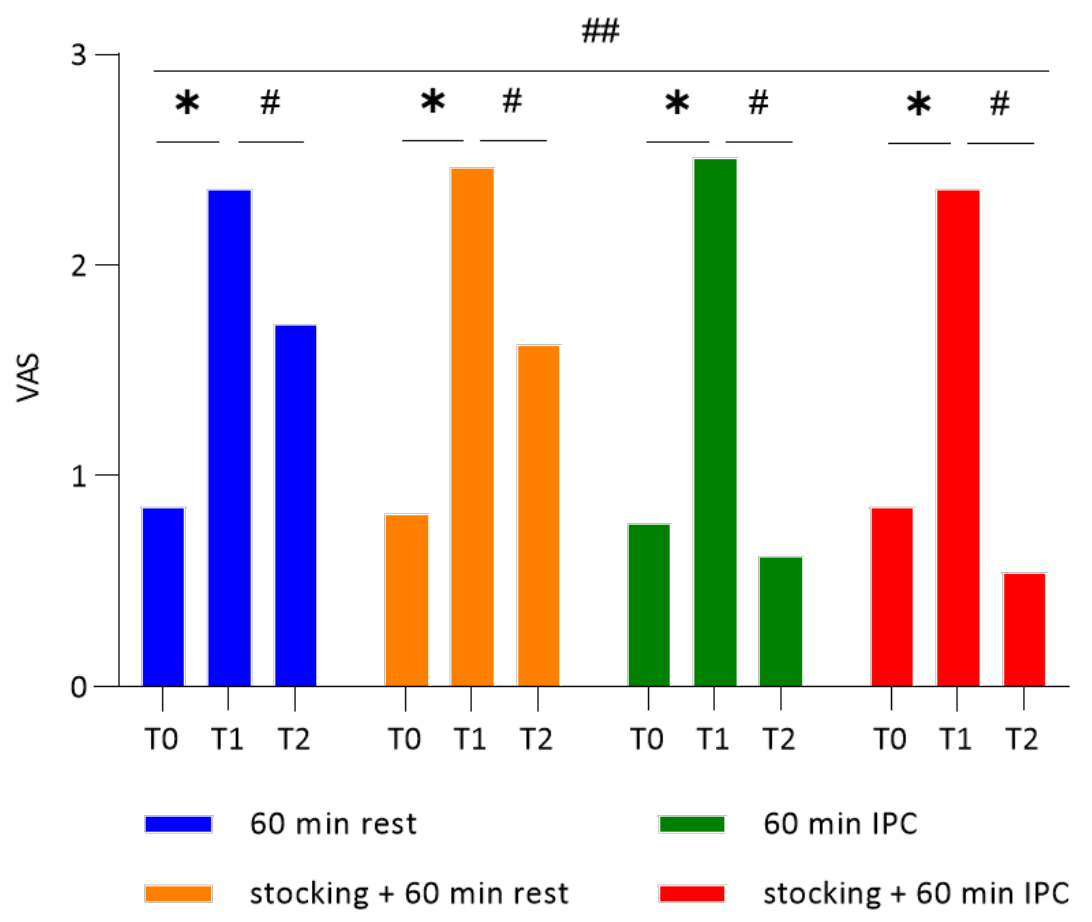

Figure 2. The effect of the different intervention groups on leg pain score (Visual Analogue Scale).

T0: before work; T1: 0 minutes after prolonged standing work; T2: 60 minutes after intervention; *: $\mathrm{p}<0.05$ between T0 and T1; \#: $\mathrm{p}<0.05$ between T1 and T2; \#\#: $\mathrm{p}<0.05$ in the four intervention groups

\subsection{Leg volume as a secondary outcome}

In the right leg, the leg volume of groups A and C, who both did not wear MCS, significantly increased in the afternoon after work (T1) compared to that measured in the morning before work (T0); meanwhile, groups B and D, who both wore MCS, showed no significant differences between T1 and T0 (Table 3, Figure 3). Despite of the prolonged standing work, the mean volume in group D decreased by $24.0 \mathrm{cc}$. The volume after interventions (T2) significantly decreased in all four groups compared to those measured at T1. In particular, group C (no MCS + IPC) showed the largest leg volume reduction of $176.9 \mathrm{cc}$. There were also significant differences across the four interventions $(p=0.014)$. In the posthoc test, the difference in leg volume reduction was only observed between $\mathrm{A}$ and $\mathrm{C}, \mathrm{B}$ and C, and C and D (Supplementary Table S2). 

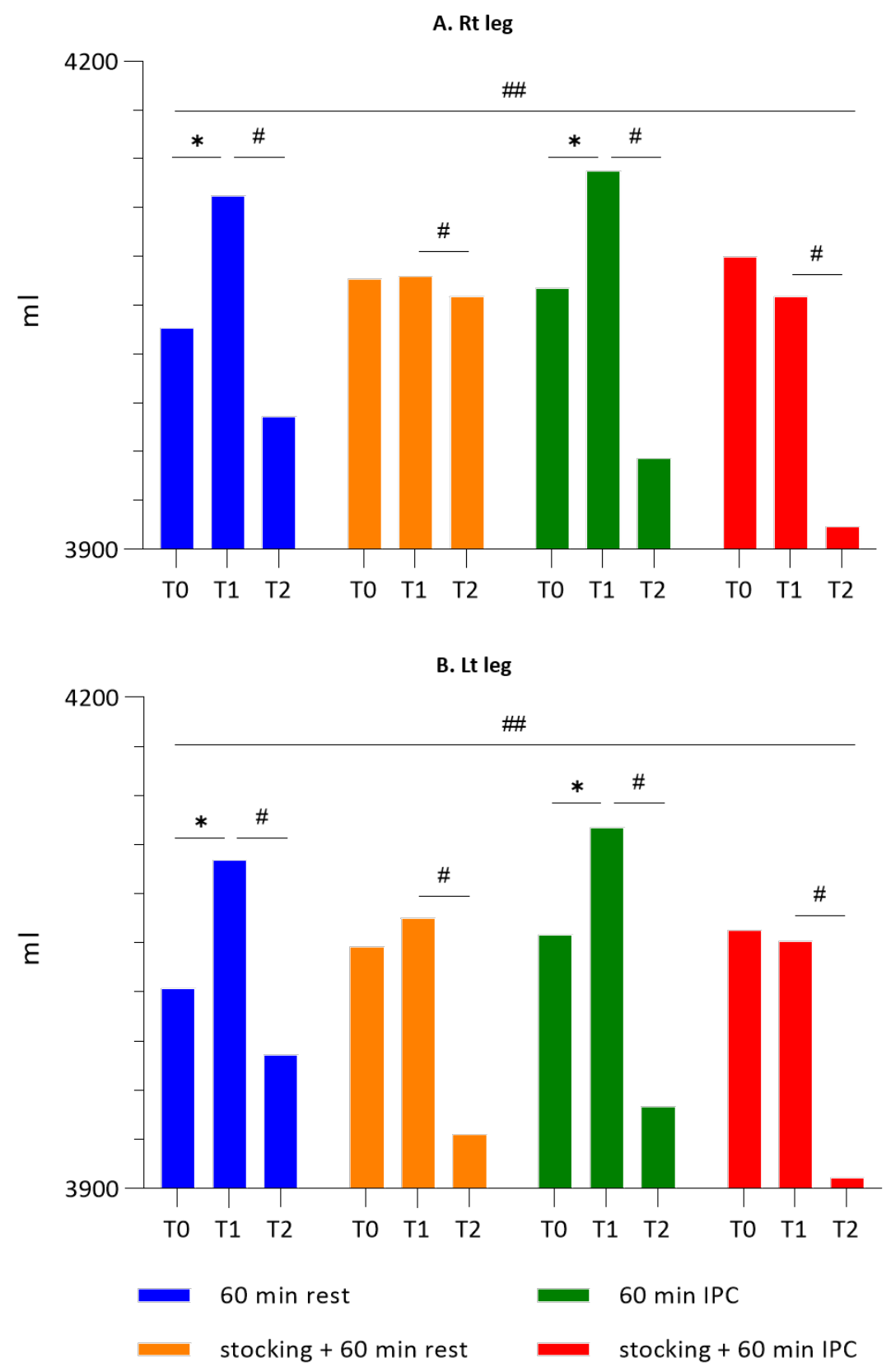

Figure 3. The effect of the different intervention groups on leg volume (mL).

A. Right leg, B. Left leg; T0: before work; T1: 0 minutes after prolonged standing work; T2: 60 minutes after intervention; *: p<0.05 between T0 and T1; \#: p<0.05 between T1 and T2; \#\#: $\mathrm{p}<0.05$ in the four intervention groups

Similar to the right leg, the differences in leg volume of the left leg between T0 and T1 significantly increased in groups A and C, but those in the MCS-wearing groups B and D did not show significance. In group D, the volume decreased by 6.7 cc in spite of prolonged standing work. The volume after the interventions (T2) in all four groups signifi- 
cantly decreased compared to that measured after the workday (T1), with the mean volume in group C showing the largest decrement of $170.7 \mathrm{cc}$. In addition to intra-group differences, intergroup differences were observed across the four groups $(p=0.001)$. The posthoc test showed that difference in leg volume were observed in groups A and C, B and C, and C and D.

\subsection{Leg circumference as a secondary outcome}

Leg circumference measurements at the foot and ankle, $10 \mathrm{~cm}$ below the knee, $10 \mathrm{~cm}$ above the knee, and $20 \mathrm{~cm}$ above the knee in MCS-wearing groups B and D were reduced in the afternoon after workday (T1) compared to those measured before work (T0), but the circumference measurements in groups $\mathrm{A}$ and $\mathrm{C}$, who did not use MCS, were similar or slightly increased after work (T0-T1) (Table 4, Figure 4). The circumference at all measured points after intervention (T1-T2) was significantly decreased in groups A, C, and D, although those in group B did not significantly decrease, with the exception of the both calf. When comparing the interventions, there were significant differences across interventions for the circumferences in most of the measured areas except the right foot. In the post-hoc test, there were differences in leg circumference in groups A and B, B and C, and B and D, at the right ankle, calf, distal thigh, and proximal thigh, and at the left foot, distal thigh, and proximal thigh. In groups $A$ and $C, B$ and $C$, and B and D, there were differences in circumference at the left calf, while only in groups B and C were there any difference at the left ankle (Supplementary Tables S3 and S4). 

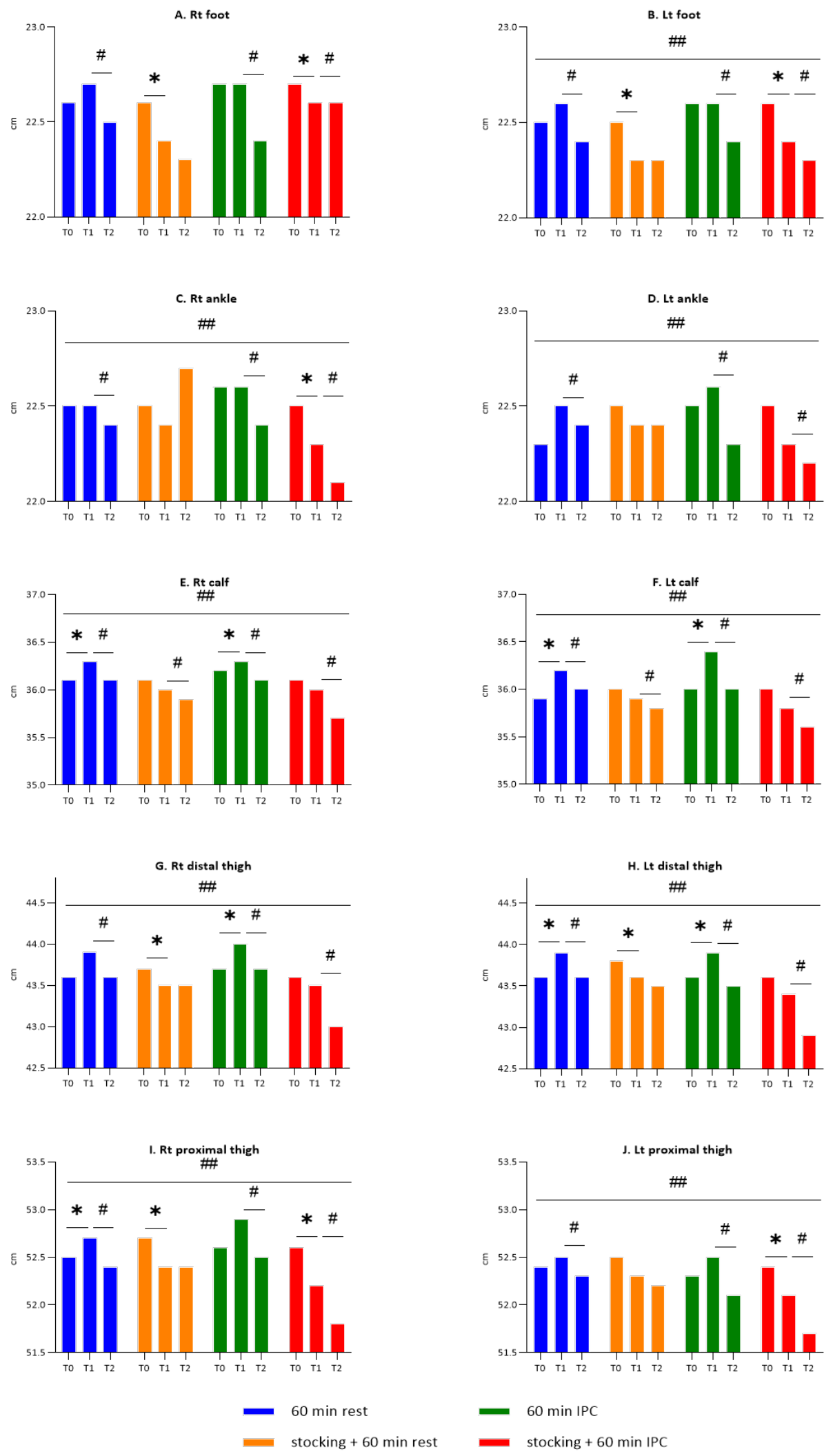

$60 \mathrm{~min}$ IPC

Figure 4. The effect of the different intervention groups on leg circumference $(\mathrm{cm})$. 
A. Right foot, B. Left foot, C. Right ankle, D. Left ankle, E. Right calf, F. Left calf, G. Right distal thigh, H. Left distal thigh, I. Right proximal thigh, J. Left proximal thigh

T0: before work; T1: 0 minutes after prolonged standing work; T2: 60 minutes after intervention; *: $\mathrm{p}<0.05$ between T0 and T1; \#: $\mathrm{p}<0.05$ between $\mathrm{T} 1$ and T2; \#\#: $\mathrm{p}<0.05$ in the four intervention groups

\subsection{Leg bioelectrical impedance analysis as a secondary outcome}

Leg ECF/TBF measured by BIA significantly decreased in all intervention groups bilaterally after the treatment session (T2) compared to those after the workday (T1) (Table 5). The ECF/TBF in group C treated with IPC showed the largest reduction, and the intergroup difference of $\mathrm{ECF} / \mathrm{TBF}$ among the four interventions was statistically significant. In the post-hoc test, there were differences observed in all groups for ECF/TBF of both legs, with the exception of groups A and D (Supplementary Table S5).

Similar to the ECF/TBF, the ECW/TBW significantly decreased in all groups bilaterally after the intervention (T1-T2), particularly in group C (Table 6). There were statistically significant differences across the interventions for ECW/TBW. Post-hoc tests showed that the differences between all paired groups, except groups A and D, significantly differed in both legs (Supplementary Table S6).

\subsection{Safety events}

Although we created a comprehensive system for monitoring and recording adverse events potentially occurring during this study, no serious adverse events were noted in the study population.

\section{Discussion}

This study confirmed that applying IPC in prolonged standing workers significantly reduced pain and swelling compared to wearing MCS. In addition, when working in a standing position all day long, there was no significant difference in pain regardless of whether or not MCS were worn. While resting supine alone or with MCS also helped improve pain, the pain improved the most when only IPC was applied, and the VAS score decreased by 1.9 points, which was found to be lower than the pain score measured in the morning. In terms of leg volume, applying IPC led to the most significant volume reduction effect among the four groups. During prolonged standing work wearing MCS for 8 $\mathrm{h}$, the leg volume was reduced immediately after working (T1), but the pain was induced similarly to when it is not worn. Leg circumference was measured at the foot, ankle, calf, distal thigh, and proximal thigh, and except for group B wearing MCS only, there was a statistically significant decrease after therapy, especially in the proximal area. Since there was no decrease in circumference nor significant difference immediately after working in group B, the effect of the therapy was relatively small compared to other groups, and there was no significant decrease except for the measurements at the both calf. The reason that there was a significant difference between group B and other groups in the post-hoc test was that there was no increase in leg circumference at T1 in group B; hence, the significant difference may appear from other groups whose leg circumference increased after working in the afternoon. ECF/TBF and ECW/TBW, which are indicators of leg edema, showed statistically significant decreases after the therapy in all groups. In particular, group C, which applied IPC alone, showed the most significant decrease. Therefore, the findings of this study confirmed that IPC can effectively treat leg pain and edema in healthy people with occupations requiring prolonged standing. Although several studies have reported on the effectiveness of IPC and MCS in healthy people standing for a long time or patients with venous insufficiency, this study contains several unique issues. First, this study targeted healthy people who had an 8-h standing occupation rather than a short standing or 
sedentary occupation. Second, Doppler ultrasound was performed as a screening test for healthy people without underlying venous disease to determine obstructive venous disease. Through this, hidden venous insufficiency was found, as well as its incidence. Third, the effectiveness of IPC and MCS was compared in healthy people and patients with venous insufficiency; previously, it was conducted only for patients with venous ulcers. Fourth, the effectiveness of each therapy on parameters and symptoms was confirmed by comparing single and combined groups of IPC and MCS. Finally, ECF/TBF and ECW/TBW were also confirmed using BIA, as well as leg swelling parameters such as volume and circumference, which have been commonly used in previous studies. In conclusion, the test is necessary if healthy people with an 8-h standing occupation have a hidden venous symptom, and this study showed that applying IPC for 30 minutes is a safe and convenient therapy to improve leg pain and swelling effectively.

A previous study of normal people with prolonged standing occupations reported that the ankle hydrostatic venous pressure increased to $90 \mathrm{mmHg}$ and leg volume deteriorated to $50 \mathrm{~mL}$ when standing for a long time [14-17]. Similar to previous studies, this study confirmed that prolonged standing work causes leg pain and swelling. In groups A and $\mathrm{C}$ where stockings were not worn, the mean leg volume at T1 compared to that at T0 increased by $65.4-81.1 \mathrm{~mL}$, and the circumference increased by $0-0.1 \mathrm{~cm}$ in the distal area below the calf and clearly increased by $0.1-0.4 \mathrm{~cm}$ in the proximal area. Prolonged standing work causes leg swelling and chronically increases hydrostatic venous pressure, leading to venous retention and venous reflux. In addition, venous reflux ultimately causes venous insufficiency, leading to secondary complications such as venous ulcers. In a previous study, venous reflux was found in more than $80 \%$ of ultrasound tests performed on healthcare workers [1]. In the duplex ultrasound screening test in this study, venous reflux was found in $89.7 \%$ of healthy people, showing results similar to the previous study. Thus, if an individual's occupation involves prolonged standing and he/she has venous symptoms in the lower limb, the risk of venous insufficiency is high and needs to be assessed.

One of the most common therapies for venous insufficiency is compression therapy, which provides graded external compression of the legs and prevents lower limb hypertension. MCS is the most commonly used compression garment, and although different pressures are recommended depending on the severity, approximately $20-50 \mathrm{mmHg}$ should be applied; wearing MCS with approximately $30-40 \mathrm{mmHg}$ is known to be helpful for pain, swelling, skin pigmentation, activity, and well-being [18]. It has been reported that wearing MCS in patients with chronic venous insufficiency significantly reduces leg pain, swelling, skin discoloration, activity tolerance, depression, and sleep problems [19]. Complete ulcer healing has been reported after wearing MCS for an average of 5.3 months in severe CVI patients with a venous ulcer [20], and a hemodynamic benefit to reduce reflux in residual volume fractional vein segments has been reported [21,22]. However, previous studies were insufficient to compare the effects of MCS and other therapies for pain and swelling, and the effects of natural resting. When comparing group A (resting) and group B (resting + MCS) in this study, the pain scores measured before and immediately after working increased in both groups, and both pain scores decreased after natural resting. However, there was no statistical difference between the two groups in the posthoc test. The volume was measured before and immediately after working, and swelling was less induced in group B than in group A. The volume measured after the intervention (T2) did not significantly differ between groups A and B at the post-hoc test. The other leg swelling indicators (circumference, ECF/TBF, and ECW/TBW) also showed similar trends. Group D, using both MCS and IPC, experienced a statistically significant effect on pain and swelling, but there was no difference with group $\mathrm{C}$ in the post-hoc test. As a result, this study showed that wearing MCS while working reduced swelling immediately after working, but there was no effect on pain reduction and swelling compared to IPC only as intervention. Additionally, the effect of the natural resting therapy tended to increase 
when wearing MCS, but it was not statistically significant, and wearing MCS did not affect IPC therapy.

IPC, which is a form of compression therapy, uses a machine that can be applied with various pressures and modes to the lower limb, and its effects and principles have been reported in several studies. According to previous studies, when IPC is applied to the feet, the peak velocity of the common femoral vein is increased; when IPC of $120-180 \mathrm{mmHg}$ is applied to the lower limb below the knee, the venous flow velocity and pulsatility index are increased. It also causes the largest increase in venous outflow in the foot and calf [23]. The risk for blood vessel obstruction with IPC use has been reported, but the application of IPC to healthy subjects increases the oxygenation of muscle cells in the lower limb [24]. Like MCS, there are still no international consensus pressure values or modes in IPC. In the evaluation of the stability and efficacy of IPC therapy in 19 healthy subjects, which was conducted before this study, a statistically significant reduction in leg pain and edema was observed when performing 30 minutes of IPC at $90-120 \mathrm{mmHg}$ and $30 \mathrm{~min}$ of natural resting, and there were no adverse effects. In addition, both the circular and sequential modes were effective without a statistical difference. Based on this, IPC intervention in this study was performed for a total of $60 \mathrm{~min}$ in circular and sequential modes for $30 \mathrm{~min}$ each at $90-120 \mathrm{mmHg}$ pressure; group $\mathrm{C}$ showed the greatest reduction in leg pain and edema. Furthermore, when observing the synergystic effect of wearing MCS during work and IPC intervention, this study showed an apparent decrease in edema immediately after working (T0-1), but there was no improvement in pain immediately after working and after the therapy (T0-1, 1-2), nor in edema after the therapy (T1-2). The effect of the compression garment was increased by maintaining pressure along with exercise or massage treatment, but the previously reported effects of MCS were not shown, since rest or exercise was avoided as much as possible and MCS was worn only in a situation where pain and swelling were induced. Alternatively, if the pressure of MCS is modified (high or low), it may be effective in reducing pain or swelling; thus, further research is needed.

This study had small numbers in each group, although significant differences were observed regarding leg pain and edema. The small numbers of the groups did not prove that there were no differences in these outcomes, but larger numbers may still not make any differences. The other limitation is that the pressure of MCS was set to $23-32 \mathrm{mmHg}$, which has reportedly been the recommended pressure. As mentioned above, the optimal setting of MCS is still controversial, and MCS intervention with a high pressure of over 33 $\mathrm{mmHg}$ might significantly reduce leg pain and edema or show a synergistic effect with IPC therapy.

\section{Conclusions}

This clinical trial suggests that IPC intervention with a pressure of $90-130 \mathrm{mmHg}$ safely and effectively reduced leg pain and edema after working in a prolonged standing position. The IPC intervention was used for a total of $60 \mathrm{~min}$, with $30 \mathrm{~min}$ of circular and sequential modes, which were based on a previous study that the effect of circular and sequential modes of IPC did not significantly affect leg pain and edema. The use of MCS also significantly decreased leg edema immediately after work, but did not show a significant difference in leg pain and edema after intervention (T1-2).

Author Contributions: Conceptualization: Y.H.W., D.S.K.; Data curation: Y.H.W.; Formal analysis: Y.H.W.; Funding acquisition: M.H.K.; Investigation: Y.H.W., D.S.K.; Methodology: Y.H.W.; Project administration: M.H.K.; Resources: M.H.K.; Software: M.H.K.; Supervision: Y.H.W., M.H.K., D.S.K.; Validation: Y.H.W., M.H.K.; Visualization: Y.H.W., D.S.K.; Writing - original draft: Y.H.W., D.S.K.; Writing - review \& editing: Y.H.W., M.H.K., D.S.K. 
Funding: This research was funded by the Korea Health Technology R\&D Project through the Korea Health Industry Development Institute (KHIDI) and by the Ministry of Health and Welfare, Republic of Korea (grant number HI15C1529.

Institutional Review Board Statement: Ethical approval for the study was obtained from the institutional review board of Jeonbuk National University Hospital (CUH 2019-05-064-002).

Informed Consent Statement: Written informed consent was obtained from all participants.

Conflicts of Interest: The authors declare no conflicts of interest.

\section{References}

1. Cires-Drouet, Rafael S., et al. 2020 High prevalence of chronic venous disease among health care workers in the United States. Journal of Vascular Surgery: Venous and Lymphatic Disorders 8(2):224-230.

2. Lastowiecka-Moras, E. Standing and sitting postures at work and symptoms of venous insufficiency - results from questionnaires and a Doppler ultrasound study. International Journal of Occupational Safety and Ergonomics.

3. Meijsen, Paul, and Hanneke J. J. Knibbe, 2007, Prolonged Standing in the OR: A Dutch Research Study. AORN Journal 86(3):399-414.

4. Waters, Thomas R., and Robert B. Dick, 2015, Evidence of Health Risks Associated with Prolonged Standing at Work and Intervention Effectiveness. Rehabilitation Nursing 40(3):148-165.

5. Mansilha, Armando, and Joel Sousa, 2018, Pathophysiological Mechanisms of Chronic Venous Disease and Implications for Venoactive Drug Therapy. International Journal of Molecular Sciences 19(6):1669.

6. Caggiati, Alberto, et al., 2018, Rehabilitation of patients with venous diseases of the lower limbs: State of the art. Phlebology: The Journal of Venous Disease 33(10):663-671.

7. Myers, Ken, and Paul Hannah, 2017, Manual of venous and lymphatic diseases: CRC Press.

8. Rabe, Eberhard, et al., 2018, Indications for medical compression stockings in venous and lymphatic disorders: An evidencebased consensus statement. Phlebology: The Journal of Venous Disease 33(3):163-184.

9. Health Guideline for standing workers. 2008; Available from:http://www.kosha.or.kr/kosha/business/musculoskeletalPreventionData_G.do?mode=view\&board $\mathrm{No}=80$ \&articleNo=296603\&attachNo=\#/list.

10. Bernas MJ, Witte MH, Witte C, Belch D, Summers P: Limb volume measurements in lymphedema: issues and standards. Lymphology 1996, 29(SUPPL.):199-201.

11. Framework L: Best practice for the management of lymphoedema-International Consensus, 2006. In.: MEP Ltd, London. Available at: www. woundsinternational. com/media/issues

12. Cha K, Chertow G, Gonzalez J, et al. Multifrequency bioelectrical impedance estimates the distribution of body water. J Appl Physiol 1995;79:1316-9.

13. Moseley A, Piller N, Carati C. Combined opto-electronic perometry and bioimpedance to measure objectively the effectiveness of a new treatment intervention for chronic secondary leg lymphedema. Lymphology 2002;35:136-43.

14. Blättler W, Thomae H-J, Amsler F. Venous leg symptoms in healthy subjects assessed during prolonged standing. J Vasc Surg Venous Lymphat Disord. 2016;4:455-462.

15. Kügler C, Strunk M, Rudofsky G. Venous pressure dynamics of the healthy human leg. J Vasc Res. 2001;38:20-29.

16. Engelberger RP, Keo HH, Blaettler W, et al. The impact of orthostatic challenge on arteriovenous hemodynamics and volume changes of the lower extremity. J Vasc Surg Venous Lymphat Disord. 2013;1:250-256.

17. Pannier F, Rabe E. Optoelectric volume measurements to demonstrate volume changes in the lower extremities during orthostasis. Int Angiol. 2010;29:395-400.

18. Eberhardt RT, Raffetto JD: Chronic venous insufficiency. Circulation 2005, 111(18):2398-2409.

19. Motykie G, Caprini J, Arcelus J, Reyna J, Overom E, Mokhtee D: Evaluation of therapeutic compression stockings in the treatment of chronic venous insufficiency. Dermatologic surgery 1999, 25(2):116-120.

20. Mayberry JC, Moneta GL, Taylor L, Porter J: Fifteen-year results of ambulatory compression therapy for chronic venous ulcers. Surgery 1991, 109(5):575-581.

21. Ibegbuna V, Delis KT, Nicolaides AN, Aina O: Effect of elastic compression stockings on venous hemodynamics during walking. Journal of vascular surgery 2003, 37(2):420-425.

22. Zajkowski PJ, Proctor MC, Wakefield TW, Bloom J, Blessing B, Greenfield LJ: Compression stockings and venous function. Archives of surgery 2002, 137(9):1064-1068.

23. Killewich LA, Sandager GP, Nguyen AH, Lilly MP, Flinn WR. Venous hemodynamics during impulse foot pumping. J Vasc Surg. 1995;22:598-605.

24. Nandwana SK, Ho KM. A comparison of different modes of pneumatic compression on muscle tissue oxygenation: An intraparticipant, randomised, controlled volunteer study. Anaesth Intens Care. 2019;47:23-31. 